\title{
Investigation of Robust Stability for Fractional-Order LTI Systems with Multilinear Structure of Ellipsoidal Parametric Uncertainty
}

\author{
Radek Matušů ${ }^{1[0000-0002-5242-7781]}$ and Bilal Șenol ${ }^{2[0000-0002-3734-8807]}$ \\ ${ }^{1}$ Centre for Security, Information and Advanced Technologies (CEBIA-Tech) \\ Faculty of Applied Informatics, Tomas Bata University in Zlín \\ nám. T. G. Masaryka 5555, 76001 Zlín, Czech Republic \\ rmatusueutb.cz \\ ${ }^{2}$ Department of Computer Engineering \\ Faculty of Engineering, Inonu University \\ 44280 Malatya, Turkey \\ bilal.senoldinonu.edu.tr
}

\begin{abstract}
The contribution focuses on the investigation of robust stability for fractional-order linear time-invariant (LTI) systems with the multilinear structure of ellipsoidal parametric uncertainty, i.e., the analyzed family of fractional-order polynomials has the multilinear uncertainty structure and an ellipsoid-shaped uncertainty bounding set. The robust stability test is based on the numerical calculation and subsequent plot of the value sets, and the application of the zero exclusion condition. Unlike the previously published works, this contribution shows that, contrary to the case of a two-dimensional ellipse of parameters, the internal points of a three-dimensional ellipsoid of parameters cannot create the boundary of the value set in the complex plane even under more complicated uncertainty structures, such as the multilinear one.
\end{abstract}

Keywords: Robust Stability, Fractional-Order Systems, Ellipsoidal Parametric Uncertainty, Multilinear Uncertainty, Value Set.

\section{Introduction}

The precise knowledge of all physical parameters is only an idealized scenario in the majority of practical control applications. Moreover, the simplifications and linearizations made during the modeling contribute also to the typical mismatch between the mathematical model and the true behavior of the controlled plant. That is why the use of uncertain models and robust control techniques has been extremely popular during the last decades.

Since stability is the critical requirement of control loops, we are interested in robust stability from the viewpoint of models with uncertainty. It means that the stability of the control system must be secured not only for one nominal plant but also for the whole group of plants, which is usually called the family of plants. A common way of defining 
such family consists in the use of parametric uncertainty $[1,2]$, i.e., the structure is "known" and fixed, but the parameters may "slowly" vary within prescribed bounds. The commonly used engineering approach is to consider these bounds as real intervals; in other words, the bound has a shape of a box. However, there are some other alternative shapes available, such as a diamond, a sphere, or, more generally, an ellipsoid [210].

The popularity of fractional-order systems and their application in control engineering has been growing rapidly [11-13] for the sake of their improved performance compared with the conventional integer-order systems. It is understandable that great research attention is also paid to the robust stability of fractional-order systems under various uncertainty conditions $[14,15]$.

The contribution deals with the investigation of robust stability for fractional-order LTI systems with the multilinear structure of ellipsoidal parametric uncertainty. In other words, the studied family of fractional-order (characteristic) polynomials has the multilinear uncertainty structure and an ellipsoid-shaped uncertainty bounding set. The robust stability analysis takes advantage of the numerical calculation and subsequent plot of the value sets and the application of the zero exclusion condition [2].

More specifically, this contribution is intended to be a follow-up to the previously published works [8-10]. In [8], the families of integer-order polynomials with multilinear uncertainty structure were studied, but the example dealt only with a two-dimensional uncertainty bounding set (ellipse). Then, in [9], the fractional-order version of the polynomial family with multilinear uncertainty structure was studied, among other structures, but only for the elliptical uncertainty bounding set as well. The case of a three-dimensional uncertainty bounding set (ellipsoid) was outlined in [9] in combination with the polynomic uncertainty structure. In [10], the three-dimensional uncertainty bounding set was combined with the independent uncertainty structure. Unlike the previous works, this contribution demonstrates that, contrary to the case of a twodimensional ellipse of parameters [8,9], the internal points from three-dimensional ellipsoid of parameters cannot create the boundary of the value set in the complex plane even under more complicated uncertainty structures, such as the multilinear uncertainty structure.

Since this contribution is example-oriented and the level of presented theory and literature survey is restricted to a minimum, the interested readers are referred to the authors' previous works, especially [9].

\section{Mathematical Description of Analyzed Family}

Suppose that a family of systems has a corresponding family of fractional-order characteristic polynomials with three uncertain parameters and that this family of fractionalorder polynomials is defined by the multilinear structure of uncertainty, according to:

$$
\begin{aligned}
& p(s, q)=s^{3.1}+\left(2 q_{1} q_{2} q_{3}+3 q_{1} q_{2}-q_{2} q_{3}\right) s^{2.2}+\cdots \\
& +\left(5 q_{1} q_{2} q_{3}+0.1 q_{1}+0.2 q_{2}+0.3 q_{3}\right) s^{0.9}+\left(q_{1} q_{2}+q_{2} q_{3}+q_{1} q_{3}\right)
\end{aligned}
$$


and by the uncertainty bounding set $Q$ in a shape of a (three-dimensional) ellipsoid, described by:

$$
\begin{aligned}
& \left\|q-q^{0}\right\|_{2, W} \leq r=1 \\
& q^{0}=\left[q_{1}^{0}, q_{2}^{0}, q_{3}^{0}\right]=[2,1,0.5] \\
& W=\left[\begin{array}{ccc}
w_{1}^{2} & 0 & 0 \\
0 & w_{2}^{2} & 0 \\
0 & 0 & w_{3}^{2}
\end{array}\right]=\left[\begin{array}{ccc}
2 & 0 & 0 \\
0 & 5 & 0 \\
0 & 0 & 10
\end{array}\right]
\end{aligned}
$$

Alternatively, the same uncertainty bounding set can be written in the form:

$$
\begin{aligned}
& 2\left(q_{1}-q_{1}^{0}\right)^{2}+5\left(q_{2}-q_{2}^{0}\right)^{2}+10\left(q_{3}-q_{3}^{0}\right)^{2} \leq r=1 \\
& q^{0}=\left[q_{1}^{0}, q_{2}^{0}, q_{3}^{0}\right]=[2,1,0.5]
\end{aligned}
$$

The graphical representation of this uncertainty bounding set, given either by (2) or (3), is depicted in Fig. 1. The axes are plotted in an equal aspect ratio. Unsurprisingly, the uncertainty bounding set really has the ellipsoidal shape, and thus, all admissible triplets of parameters $q_{1}, q_{2}$, and $q_{3}$ have to be located inside or on the surface of this ellipsoid.

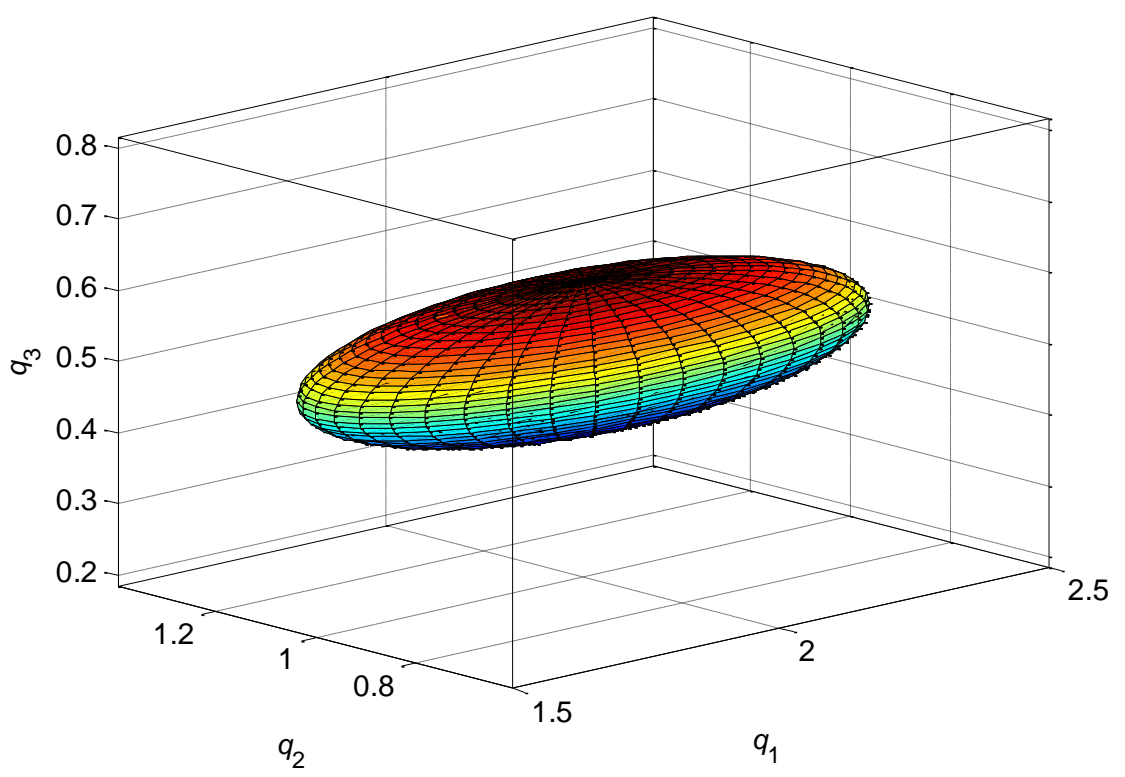

Fig. 1. Ellipsoidal uncertainty bounding set (2), (3). 


\section{Value Set - Construction and Properties}

The applied graphical test of robust stability is based on plotting the value sets and verifying the condition for zero exclusion. Therefore, a discussion on the value set of the family (1), (2) follows.

The value set $p(j \omega, Q)=\{p(j \omega, q): q \in Q\}$ [2] for a chosen fixed frequency $\omega=2$ and various "gridding" is shown in Figs. 2-4. As can be seen in [8, 9], in the case of multilinear or more complicated uncertainty structure and a two-dimensional uncertainty bounding set, the boundaries of the value set in the complex plane may be mapped not only from the boundaries in two-dimensional parameter space (ellipse) but possibly also from some internal points. This holds true both for the integer-order [8] and for the fractional-order [9] polynomials. However, for the case of a three-dimensional uncertainty bounding set, it is enough to grid just the surface area of the ellipsoid, because no internal point from the ellipsoid of parameters (Fig. 1) may be mapped to the boundary of the value set. In other words, no internal points can comprise the value set boundary. Contrary to the statement in [10], this holds true not only for simple uncertainty structures but even for the complicated ones (such as multilinear uncertainty structure in this paper).

Thus, the surface area of the ellipsoid from Fig. 1 will be gridded, and subsequently, the related value set points will be directly computed and plotted. The surface area can be parametrized, e.g., by using the set of parametric equations:

$$
\begin{aligned}
& q_{1}=\left(\frac{r}{w_{1}^{2}}\right) \cos \theta \cos \varphi+q_{1}^{0} \\
& q_{2}=\left(\frac{r}{w_{2}^{2}}\right) \cos \theta \sin \varphi+q_{2}^{0} \\
& q_{3}=\left(\frac{r}{w_{3}^{2}}\right) \sin \theta+q_{3}^{0}
\end{aligned}
$$

where $\theta \in[0,2 \pi]$ and $\varphi \in[0, \pi]$.

First, both parameters $\theta$ and $\varphi$ were sampled by 90 equidistant samples within the whole range of aforesaid intervals. The value set for $\omega=2$ and this, relatively sparse, gridding can be seen in Fig. 2.

Next, the number of equidistant samples of $\theta$ and $\varphi$ was tripled, i.e., 270 samples within given intervals are used. The resulting value set (again for $\omega=2$ ) is shown in Fig. 3. Obviously, it is much denser compared to the value set from Fig. 2. However, the cost for it is a higher computational effort. 


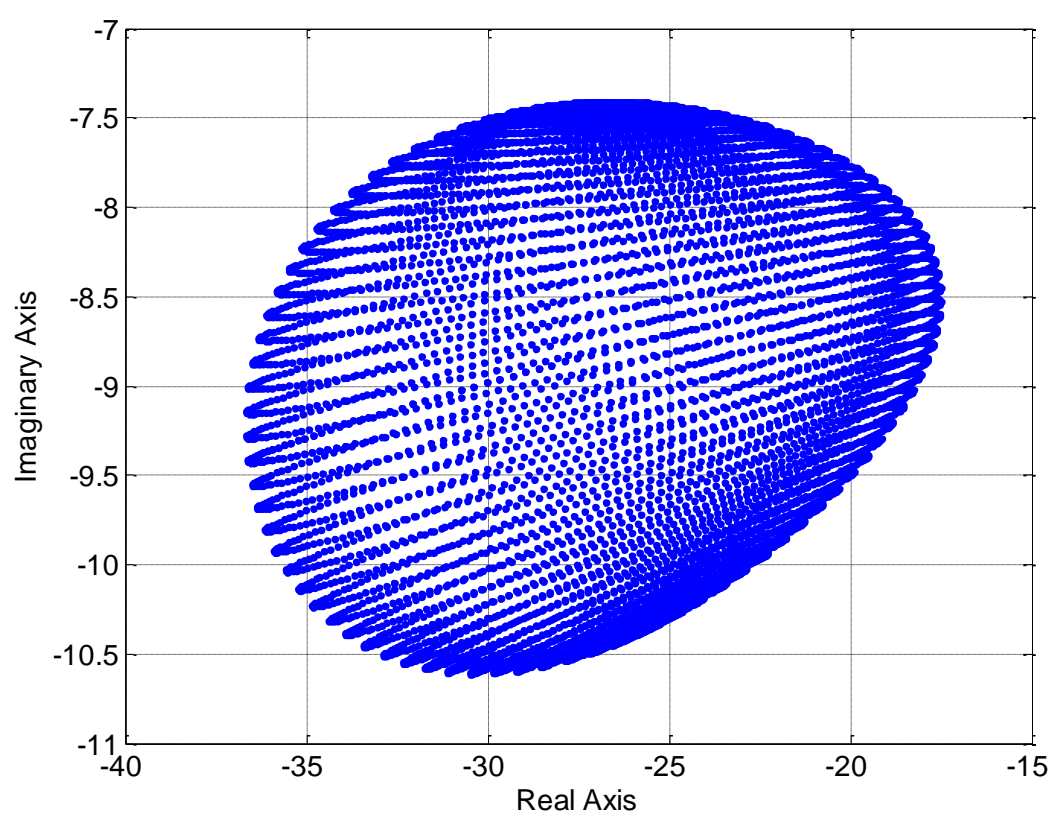

Fig. 2. Value set of family (1), (2) at $\omega=2$ for 90 samples of both $\theta$ and $\varphi$

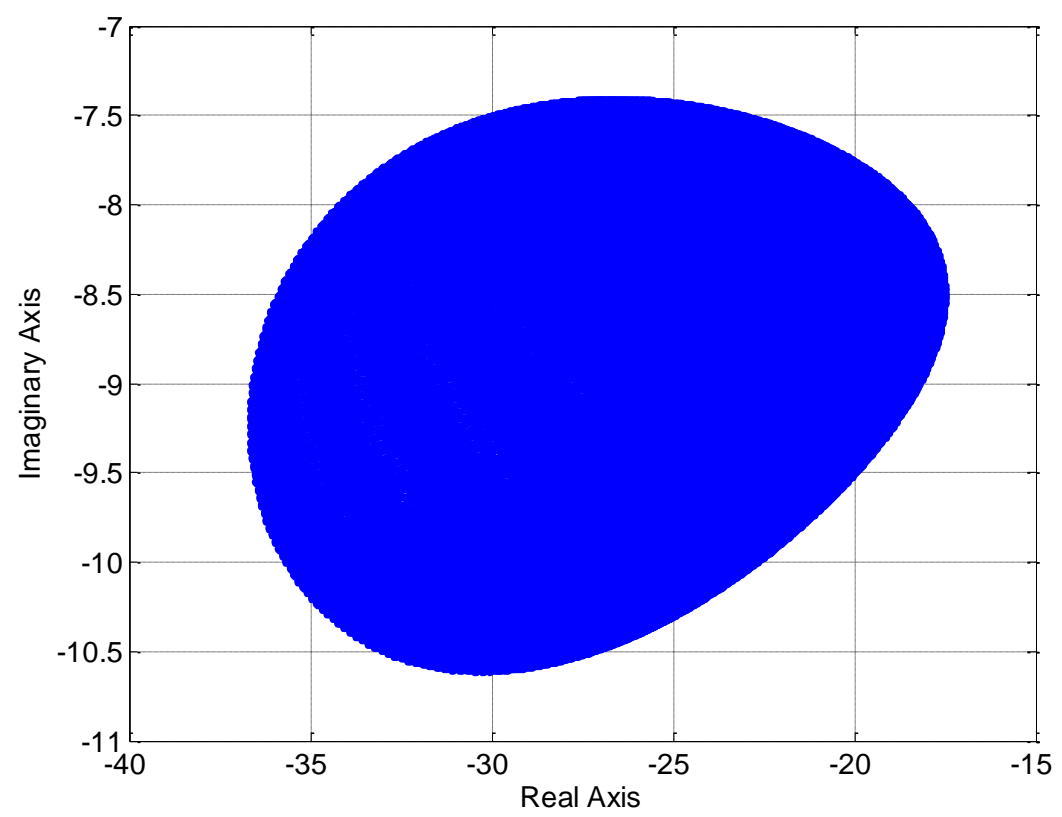

Fig. 3. Value set of family (1), (2) at $\omega=2$ for 270 samples of both $\theta$ and $\varphi$ 
Note that some points from the surface area of the three-dimensional ellipsoid of parameters (Fig. 1) are mapped to the boundary of the value set in the complex plane while the others are mapped to the interior of the value set (Figs. 2 and 3). Nevertheless, as discussed above, no internal point from the three-dimensional ellipsoid of parameters can be mapped to the boundary of the value set, even for the family of fractional-order polynomials with multilinear uncertainty structure. For illustration, Fig. 4 presents the same value set as in Fig. 3, but it also contains 10,000 extra points (red x-marks) that are mapped from 10,000 randomly chosen points from the interior of the ellipsoid of parameters.

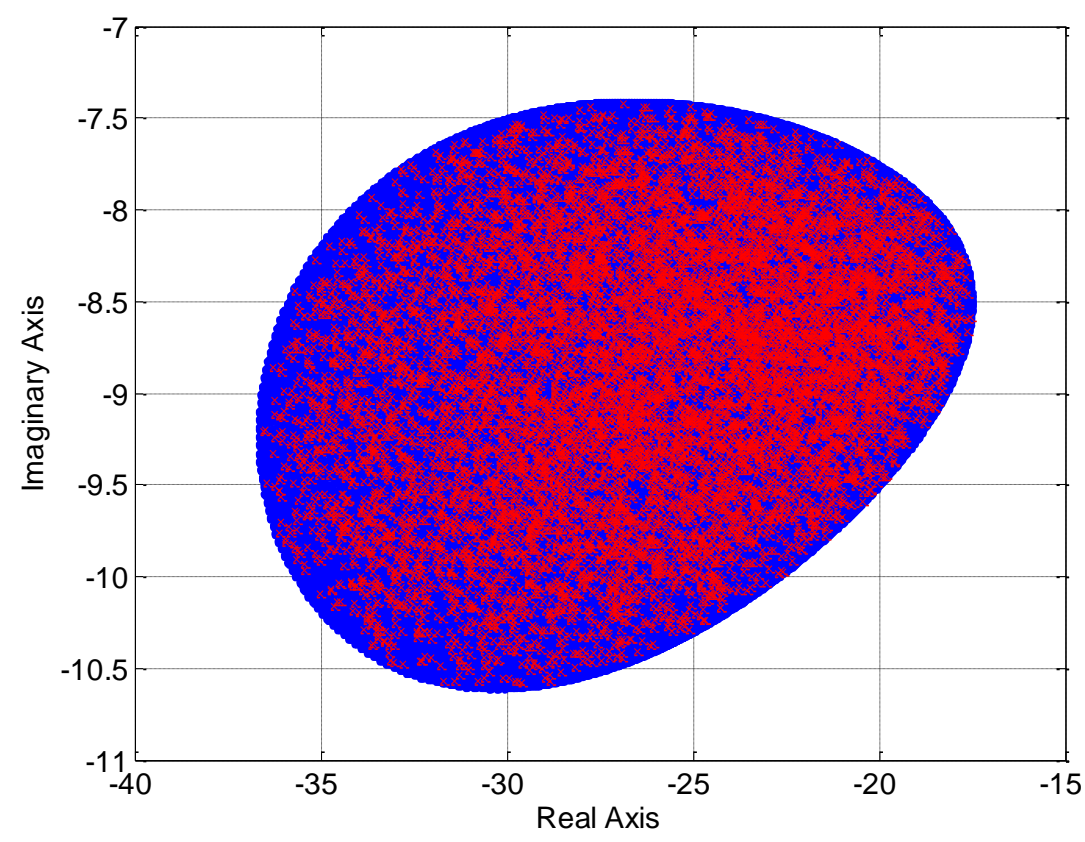

Fig. 4. Value set of family (1), (2) at $\omega=2$ with 10,000 extra random points

As can be seen, Fig. 4 is not much convincing, because some red points lie very close to the value set boundary, even though not in the boundary itself. Thus, the procedure is repeated, but 10,000 random points are taken from the inner space of the ellipsoid according to:

$$
2\left(q_{1}-2\right)^{2}+5\left(q_{2}-1\right)^{2}+10\left(q_{3}-0.5\right)^{2} \leq 0.9
$$

The value set with 10,000 extra points (red x-marks) mapped from 10,000 randomly selected points in the parameter space that comply with (5) is depicted in Fig. 5. It demonstrates that, contrary to the case of a two-dimensional ellipse of parameters [8, 9], the internal points from a three-dimensional ellipsoid of parameters cannot create 
the boundary of the value set even under more complicated uncertainty structures, such as the multilinear one.

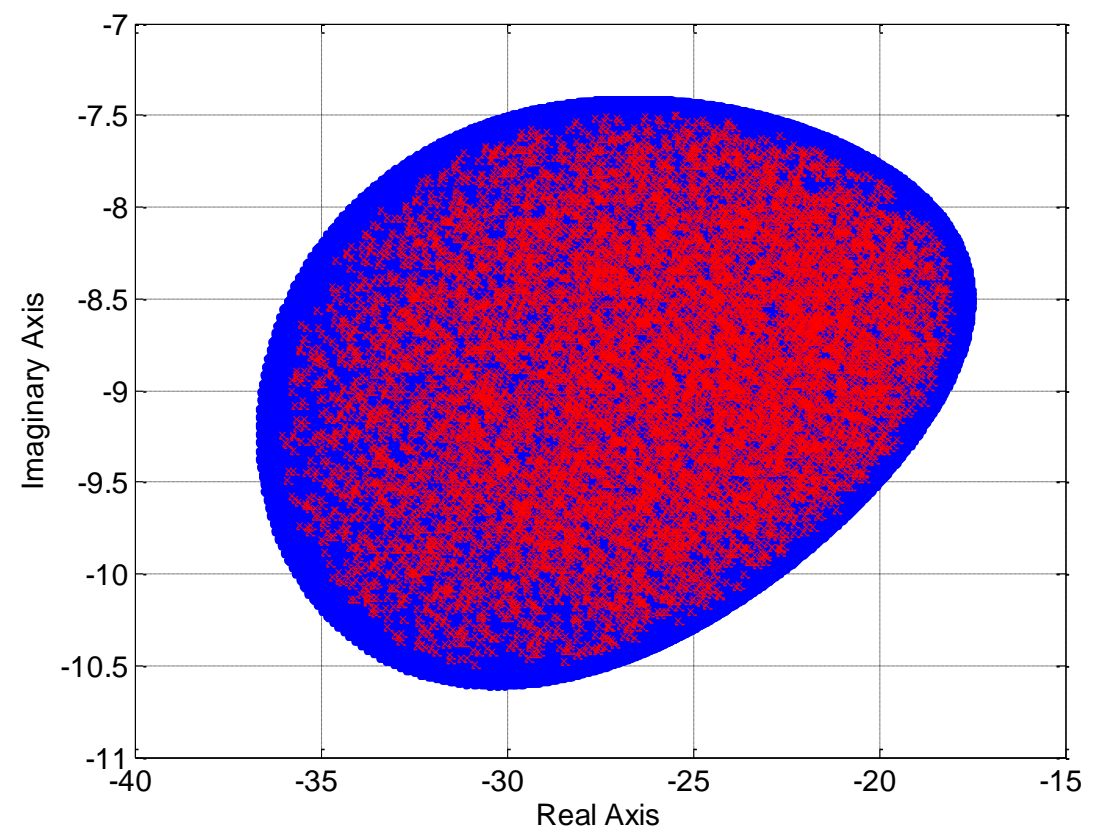

Fig. 5. Value set of family (1), (2) at $\omega=2$ with 10,000 extra random points that fulfill (5)

\section{Robust Stability Test}

In the final phase, the value sets will be utilized for the simple graphical test of robust stability. The procedure of obtaining the value set at one fixed frequency, e.g., $\omega=2$, and for 270 samples of $\theta$ and $\varphi$ (Fig. 3) will be repeated for a selected range of nonnegative frequencies. The Fig. 6 shows the value sets for the range of frequencies from 0 to 2.5 with the step size 0.1 The value sets would enter the fourth quadrant for even higher frequencies, but its current range is selected in order to have a good view of the most important area, which is the neighborhood of the zero (complex plane origin).

In accordance with the zero exclusion condition [2,9], the family of fractional-order polynomials with the multilinear structure of ellipsoidal parametric uncertainty (1), (2) is robustly stable because the zero point is excluded from the value sets and all necessary preconditions (invariant degree of the polynomial family, pathwise connected $Q$, continuous coefficient functions, and at least one stable member of the family) are fulfilled. 


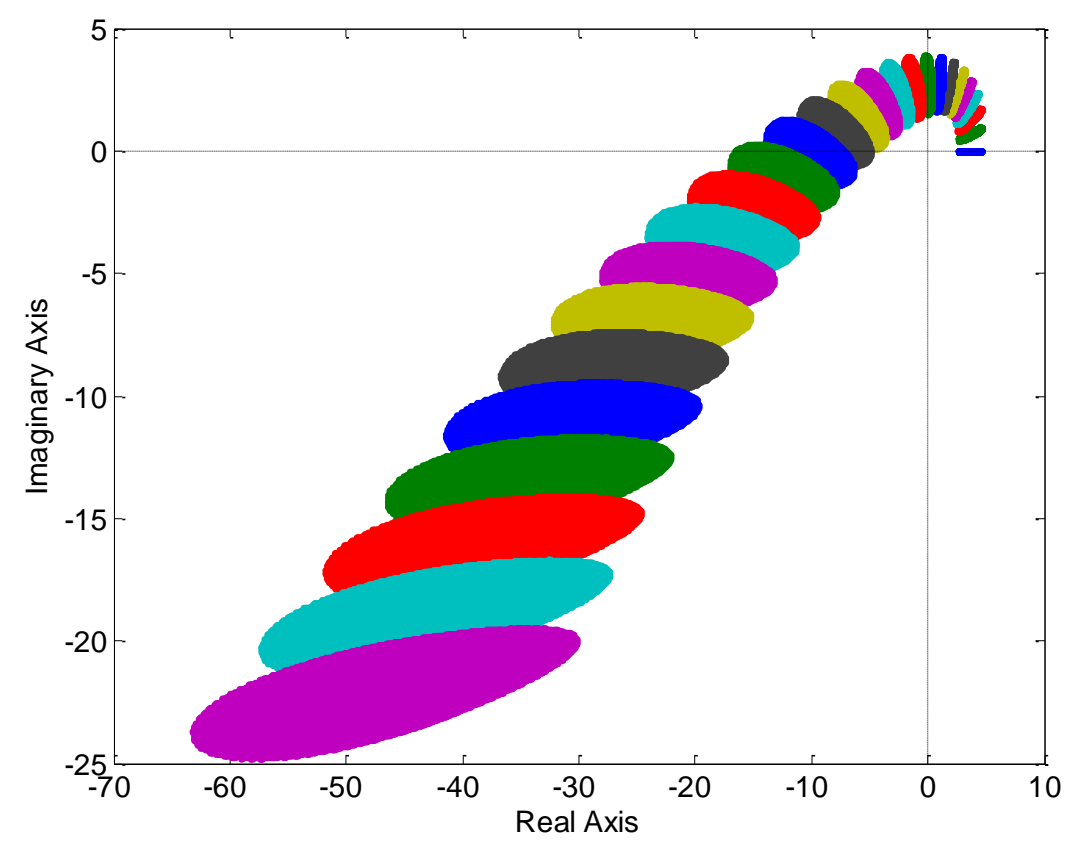

Fig. 6. Value sets of family (1), (2) for $\omega=0: 0.1: 2.5$

\section{Conclusion}

This example-oriented contribution was focused on graphical testing of robust stability for fractional-order LTI systems with the multilinear structure of ellipsoidal parametric uncertainty via the numerical calculation of the value sets, and the use of the zero exclusion principle. It is intended as a follow-up to some previously published works. Unlike them, this contribution showed that the internal points of a three-dimensional ellipsoid of parameters could not produce the boundary of the value set even under more complicated uncertainty structures, such as the multilinear one, which is not true for the case of a two-dimensional ellipse of parameters.

Acknowledgments. This work was supported by the Ministry of Education, Youth and Sports of the Czech Republic within the National Sustainability Programme project No. LO1303 (MSMT-7778/2014).

\section{References}

1. Bhattacharyya, S.P.: Robust control under parametric uncertainty: An overview and recent results. Annual Reviews in Control, 44, 45-77 (2017). 
2. Barmish, B.R.: New Tools for Robustness of Linear Systems. Macmillan, New York, USA (1994).

3. Sadeghzadeh, A., Momeni, H., Karimi, A.: Fixed-order $\mathrm{H}_{\infty}$ controller design for systems with ellipsoidal parametric uncertainty. International Journal of Control, 84(1), 57-65 (2011).

4. Sadeghzadeh, A.: Identification and robust control for systems with ellipsoidal parametric uncertainty by convex optimization. Asian Journal of Control, 14(5), 1251-1261 (2012).

5. Sadeghzadeh, A., Momeni, H.: Robust output feedback control for discrete-time systems with ellipsoidal uncertainty. IMA Journal of Mathematical Control and Information, 33(4), 911-932 (2016).

6. Matušů, R.: Spherical Families of Polynomials: A Graphical Approach to Robust Stability Analysis. International Journal of Circuits, Systems and Signal Processing, 10, 326-332 (2016).

7. Matušů, R.: Value Sets of Ellipsoidal Polynomial Families with Affine Linear Uncertainty Structure. In: Cybernetics and Automation Control Theory Methods in Intelligent Algorithms: Proceedings of 8th Computer Science On-line Conference 2019, Vol. 3 - Advances in Intelligent Systems and Computing, vol. 986, pp. 255-263, Springer Nature Switzerland AG, Cham (2019).

8. Matušủ, R., Şenol, B.: Application of Value Set Concept to Ellipsoidal Polynomial Families with Multilinear Uncertainty Structure. In: Computational Statistics and Mathematical Modeling Methods in Intelligent Systems: Proceedings of 3rd Computational Methods in Systems and Software 2019, Vol. 2 - Advances in Intelligent Systems and Computing, vol. 1047, pp. 81-89, Springer Nature Switzerland AG, Cham (2019).

9. Matušů, R., Şenol, B., Pekař, L.: Value-Set-Based Approach to Robust Stability Analysis for Ellipsoidal Families of Fractional-Order Polynomials with Complicated Uncertainty Structure. Applied Sciences, 9(24), Article No. 5451 (2019).

10. Matušů, R., Şenol, B., Vašek, V.: Robust Stability of Fractional-Order LTI Systems with Independent Structure of Ellipsoidal Parametric Uncertainty. In: Proceedings of the 30th DAAAM International Symposium, pp. 161-166, Vienna, Austria (2019).

11. Machado, J.A.T., Kiryakova, V., Mainardi, F.: Recent history of fractional calculus. Communications in Nonlinear Science and Numerical Simulation, 16(3), 1140-1153 (2011).

12. Chen, Y., Petráš, I., Xue, D.: Fractional Order Control - A Tutorial, In: Proceedings of the 2009 American Control Conference, St. Louis, MO, USA (2009).

13. Xue, D.: Fractional-Order Control Systems: Fundamentals and Numerical Implementations. De Gruyter, Berlin, Germany (2017).

14. Tan, N., Özgüven, Ö.F., Özyetkin, M.M.: Robust stability analysis of fractional order interval polynomials. ISA Transactions, 48(2), 166-172 (2009).

15. Jiao, Z., Zhong, Y.: Robust stability for fractional-order systems with structured and unstructured uncertainties. Computers \& Mathematics with Applications, 64(10), 3258-3266 (2012). 